\title{
A Transgenic Mutant of Lactuca sativa (Lettuce) with a T-DNA Tightly Linked to Loss of Downy Mildew Resistance
}

\author{
Patricia A. Okubara, Rosa Arroyo-Garcia, Katherine A. Shen, Marianne Mazier, Blake C. Meyers, \\ Oswaldo E. Ochoa, Shinje Kim, Chang-Hsien Yang, and Richard W. Michelmore \\ Department of Vegetable Crops, University of California, Davis 95616, U.S.A. \\ Accepted 14 August 1997.
}

\begin{abstract}
One hundred and ninety-two independent primary transformants of lettuce cv. Diana were obtained by co-cultivation with Agrobacterium tumefaciens carrying constructs containing maize $A c$ transposase and $D s . \mathbf{R}_{2}$ families were screened for mutations at four genes $(\mathrm{Dm})$ for resistance to downy mildew. One family, designated dm3t524, had lost resistance to an isolate of Bremia lactucae expressing the avirulence gene Avr3. Loss of resistance segregated as a single recessive allele of $\mathrm{Dm} 3$. The mutation was not due to a large deletion as all molecular markers flanking $\mathrm{Dm} 3$ were present. Loss of $\mathrm{Dm} 3$ activity co-segregated with a TDNA from which $D s$ had excised. Genomic DNA flanking the right border of this T-DNA was isolated by inverse polymerase chain reaction. This genomic sequence was present in four to five copies in wild-type cv. Diana. One copy was missing in all eight deletion mutants of $\mathrm{Dm} 3$ and altered in dm3t524, indicating tight physical linkage to Dm3. Three open reading frames (ORFs) occurred in a 6.6-kb region flanking the insertion site; however, expression of these ORFs was not detected. No similarities were detected between these ORFs and resistance genes cloned from other species. Transgenic complementation with 11to 27-kb genomic fragments of Diana spanning the insertion site failed to restore $D m 3$ function to two ethyl methanesulfonate (EMS)-induced mutants of $\mathrm{Dm} 3$ or to cv. Cobham Green, which naturally lacks Dm3 activity. Therefore, either the T-DNA inserted extremely close to, but not within, $D m 3$ and the mutation may have been caused by secondary movement of $D s$, or $D m 3$ activity is encoded by a gene extending beyond the fragments used for complementation.
\end{abstract}

Corresponding author: Richard W. Michelmore

E-mail: rwmichelmore@ucdavis.edu

Present address of Patricia A. Okubara: USDA-ARS/WRRC, $800 \mathrm{Bu}-$ chanan Street, Albany, CA 94710, U.S.A.

Present address of Marianne Mazier: INRA Station d'Amelioration des Plantes Maraicheres, BP94, F84143 Montfavet Cedex, France.

Present address of Chang-Hsien Yang: Institute of Agricultural Biotechnologies, National Chung Hsing University, Taichung, Taiwan 40227, Republic of China.

Nucleotide and/or amino acid sequence data are to be found at GenBank as accession numbers AF018069 and AF018070.
Additional keywords: disease resistance.

At least 10 different resistance genes have been isolated from several species by map-based cloning or transposon tagging (reviewed in Staskawicz et al. 1995; Bent 1996). Mutants have played critical roles in the cloning of many of these genes, either as part of a transposon tagging strategy: HMI from corn for resistance to Cochliobolus heterostrophus (Johal and Briggs 1992), $N$ from tobacco conferring resistance to tobacco mosaic virus (Whitham et al. 1994), Cf9 for resistance to Cladosporium fulvum in tomato (Jones et al. 1994), and L6 encoding resistance to rust in flax (Lawrence et al. 1995) - or for initial definition of the locus as well as confirmation of genes isolated by map-based cloning: Prf required for resistance to Pseudomonas syringae and sensitivity to the insecticide fenthion in tomato (Salmeron et al. 1994), and RPS2 for resistance to Pseudomonas syringae in Arabidopsis thaliana (Bent et al. 1994). Similarities between cloned resistance genes is facilitating the isolation of additional resistance genes as candidate sequences can be recognized (Kanazin et al. 1996; Yu et al. 1996; reviewed in Michelmore 1995a). The deduced amino acid sequences of Prf, RPS2, RPM1, N, and others each have a conserved nucleotide-binding site (Grant et al. 1995). Pto and Xa21 both contain motifs conserved in serine-threonine protein kinases (Martin et al. 1993; Song et al. 1995). All, except Pto and HMl, contain leucine-rich repeats that are reported to have a role in protein-protein interactions in other systems (Kobe and Deisenhofer 1994). The cloning of these genes is advancing our understanding of the molecular basis of resistance to diverse pathogens (reviewed in Bent 1996) as well as providing tools for crop improvement (reviewed in Michelmore 1995b).

We are cloning genes for resistance to downy mildew $(\mathrm{Dm})$, with an initial focus on $D m 3$, to understand the molecular basis for resistance to Bremia lactucae in lettuce. Dm genes are located in four major clusters in the lettuce genome. $D m 3$ is a member of the largest cluster that contains at least nine $D m$ genes as well as a gene for resistance to root aphid (Farrara et al. 1987). A detailed genetic map of the largest cluster has been constructed (K. Nakahara, R. Kessel, and R. Michelmore, unpublished). Deletion mutants of Dm3 were utilized to identify new molecular markers and to construct a fineresolution, deletion-breakpoint point map of the $D m 3$ region (Okubara et al. 1994; Anderson et al. 1996). Markers unique 
to the region are duplicated either closely around $\mathrm{Dm} 3$ or distributed through the resistance gene cluster. We have also generated ethyl methanesulfonate (EMS)-induced mutants of Dm genes (R. Arroyo-Garcia, D. Chin, O. Ochoa, and R. Michelmore, unpublished). Our efforts have been primarily focused on map-based cloning of $D m$ genes because the low rate of $A c$ transposition in lettuce made a transposon tagging approach impractical (Yang et al 1993a, 1993b). However, as part of our studies on the feasibility of transposon tagging of $\mathrm{Dm}$ mutants, we generated numerous transgenic families. To identify additional mutants, we screened 192 independent $R_{2}$ transgenic families for loss of activity at each of four $\mathrm{Dm}$ genes. In this paper, we report the identification and characterization of a mutant of $D m 3$ with a T-DNA inserted physically in or very close to Dm3.

\section{RESULTS}

\section{Identification and genetic analyses of a mutant allelic with $D m 3$.}

One hundred and ninety-two independent $\mathrm{R}_{2}$ families, derived from self pollination of original transformed regenerants of Lactuca sativa cv. Diana, were screened for loss of activity at four different $D m$ loci. One transgenic line, designated dm3t524, showed loss of Dm3 activity. Segregation of resistance was analyzed in $\mathrm{R}_{2}$ and $\mathrm{R}_{3}$ mutant families with $B$. lactucae isolate IM25P11. The numbers of resistant (25) and susceptible (7) $R_{2}$ seedlings did not deviate significantly from a $3: 1$ ratio expected for a recessive mutation of a single dominant gene $\left(\chi^{2} 3: 1=\right.$ $0.17)$. A total of $55 \mathrm{R}_{3}$ seedlings from two different susceptible $R_{2}$ individuals were entirely susceptible to IM25P11, consistent with a homozygous recessive mutation.

Allelism tests were made by crossing homozygous dm3t524 individuals with a total of 15 other Dm3 mutants, eight deletion mutants, two spontaneous mutants, and five EMS mutants. From 10 to $55 \mathrm{~F}_{1}$ progeny were pooled from reciprocal crosses with each $D m 3$ mutant. $F_{1}$ progeny from all crosses were susceptible to IM25P11, indicating that the mutation in dm3t524 was allelic to the other $15 \mathrm{Dm} 3$ mutations tested. Progeny of crosses with the deletion mutants could still show loss of Dm3 activity if two or more closely linked genes were required for $D m 3$ activity and both genes were missing in the deletion mutants. However, crosses between dm3t524 and the five independent EMS mutants, all of which do not lack any flanking markers and are therefore likely to carry point mutations, resulted in no additional complementation groups. Therefore, there appears to be only a single mutable gene conferring $D m 3$ specificity and the mutation in dm3t524 is allelic to $D m 3$.

\section{Loss of resistance is correlated to the presence of the pSLJ3621 T-DNA.}

The segregation of the $A c$ transposase, $D s$, and the $D s$ empty donor site in the spectinomycin resistance gene (AadA) was tested by polymerase chain reaction (PCR). PCR analysis confirmed the earlier observation in which spectinomycin insensitivity segregated 3:1 (20 uniformly green : 6 uniformly white) when $R_{2}$ seedlings were tested on plates containing spectinomycin; no green-white chimeras were observed (Yang et al. 1993a). Therefore, excision of $D s$ and the consequent restoration of a functional $A a d A$ gene had occurred early in the transformation process, prior to the regeneration of the $\mathrm{R}_{1}$ plant of dm3t524. Ds was not detectable in either Dm3resistant or $D m 3$-susceptible plants by PCR with $D s$-specific primers, indicating that $D s$ had not reintegrated after excision or been lost by somatic segregation. As expected, $16 \mathrm{R}_{2}$ plants that showed no sporulation with IM25P11 (either homozygous or heterozygous for $D m 3$ ) were segregating for both the transposase and the empty $D s$ donor site.

However, of $16 \mathrm{R}_{2}$ plants showing susceptibility to isolate IM25P11, all contained an empty $D s$ donor site, as indicated by the presence of a 580-bp PCR product amplified from the Nos promoter and a portion of the AadA gene. Only 13 of these individuals carried the $A c$ transposase. Therefore, loss of Dm3 resistance co-segregated in this limited population with an empty donor site from pSLJ3621 but not with the transposase from pSLJ3232. Genetic analyses of larger $R_{2}$ and $R_{3}$ populations was not pursued because concurrent molecular studies rendered additional segregation data unnecessary.

\section{Incomplete T-DNA insertion in dm3t524 occurred into a low copy sequence in the $\mathrm{Dm} 3$ region.}

We isolated sequences flanking the T-DNA from pSLJ3621 by inverse PCR (IPCR). IPCR with primers specific to the right T-DNA border (Table 1) resulted in the longest product of approximately $1,200 \mathrm{bp}$, designated $\mathrm{IPCR}_{1200}$, that included $800 \mathrm{bp}$ of lettuce DNA (designated $\mathrm{IPCR}_{800}$; hatched box in Figure 1). PCR products unique to dm3t524 were not obtained with primers for the left T-DNA border, suggesting that normal insertion of the left border of the T-DNA had not occurred.

The $\mathrm{IPCR}_{1200}$ fragment was used as a probe in Southern blots of wild-type cv. Diana and dm3t524 DNA (Fig. 2A). In Diana, single $4.5-\mathrm{kb}$ EcoRI or $6.0-\mathrm{kb}$ HindIII + NotI bands were observed; in dm3t524, additional bands of $8.0 \mathrm{~kb}$ (with EcoRI) and $9.5 \mathrm{~kb}$ (with HindIII + Not I) were detected. The presence in dm3t524 of more than one genomic fragment hybridizing to $\mathrm{IPCR}_{1200}$ suggested that the T-DNA had inserted into one copy of a multi-copy sequence. The additional band in each digest was only $3.5 \mathrm{~kb}$ larger, rather than the $7.2 \mathrm{~kb}$ expected for insertion of a full-length copy of the T-DNA of pSLJ3621 from which $D s$ had excised. Neither EcoRI nor HindIII + NotI cleaves within this T-DNA. The size disparity suggested that the insertion of the T-DNA was either incomplete, or was complete but accompanied by a deletion of lettuce genomic DNA. Hybridization with T-DNA sequences demonstrated that the T-DNA was only present in the larger EcoRI and HindIII + NotI bands of dm3t524 (Fig. 2B).

A variety of endonucleases were tested for their ability to resolve multiple members of the $\mathrm{IPCR}_{800}$ sequence. Digestion of DNA from Diana with one enzyme, AccI, detected multiple copies of the IPCR $_{800}$ sequence, confirming the presence of a small family of sequences (Fig. 3). At least four major AccI bands were seen in cv. Diana. A 4.8-kb fragment (Fig. 3, band 3) was absent in all of the $D m 3$ deletion mutants tested. dm3t524 also lacked this band and instead showed two novel bands of 2.6 and $2.3 \mathrm{~kb}$, explained by the presence of an AccI site close to the right border of the T-DNA. Deletion breakpoint analysis showed that the $\mathrm{IPCR}_{800}$ family members were distributed between molecular markers known to be tightly linked to Dm3 (Anderson et al. 1996). The two Dm3 mutants with the largest deletions lacked all detectable copies of 
IPCR $_{800}$. Therefore, this sequence family is tightly clustered around Dm3. We have screened approximately 65,000 amplified fragment length polymorphism (AFLP) and random amplified polymorphic DNA (RAPD) markers against the deletion panel; only one marker was missing in all deletion mutants of Dm3 (D. Lavelle, unpublished). Assuming random distribution of markers through the lettuce genome, the average spacing between the markers screened is $42 \mathrm{~kb}$. Therefore, the copy of $\mathrm{IPCR}_{800}$ on the $4.8-\mathrm{kb}$ AccI fragment is likely to be physically close to Dm3.

The number of copies of the $\mathrm{IPCR}_{800}$ sequence in the lettuce genome was also estimated by measuring the relative intensities of hybridization with a phosphoimager. DNA of dm3t524 was digested with either EcoRI or HindIII and hybridized to $660 \mathrm{bp}$ of lettuce DNA from the IPCR $_{1200}$ fragment. Amounts of radiolabeled probe hybridizing to both the T-DNA tagged copy or the faster-migrating untagged copy(s) were quantified with a phosphoimager linked to MACBAS 2.0. Twofold more radiolabel was measured in the smaller EcoRI band than in the larger, T-DNA-containing band, and two- to threefold more radiolabel was measured in the smaller HindIII band. Assuming that the T-DNA-tagged band was single copy, we estimated three to four copies of the $\mathrm{IPCR}_{800}$ sequence in $\mathrm{dm} 3 \mathrm{t} 524$. This is consistent with Southern blots of AccIdigested DNA (Fig. 3) that indicated four to five different fragments hybridizing to the $\mathrm{IPCR}_{800}$ sequence.

\section{Characterization of BAC clones carrying the IPCR sequence.}

In a screen of over 50,000 bacterial artificial chromosome (BAC) clones, representing two genome equivalents of lettuce, four BAC clones, designated BACH15, BACH18, BACH19, and BACE42, hybridized to the 660-bp fragment from IPCR 1200 . All of the clones carried a 4.0-kb AccI fragment; none carried the 4.8-kb fragment that was missing or altered in the $\mathrm{Dm} 3 \mathrm{mu}-$ tants. It was surprising that all four of the BAC clones only contained the smallest AccI fragment seen in genomic Southern blots of wild-type Diana and Dm3 mutants. The pattern of IPCR $_{800}$-containing fragments in genomic Southern blots might be attributed to differential digestion of genomic DNA by AccI, which recognizes the degenerate palindromic sequence GTA/CG/TAC and is subject to site-dependent deoxyguanosine methylation (e.g., Webb and Chaplin 1990). Such methylation would not occur on plasmid DNA replicated in Escherichia coli. Therefore, the AccI digestion pattern of the BAC clones did not

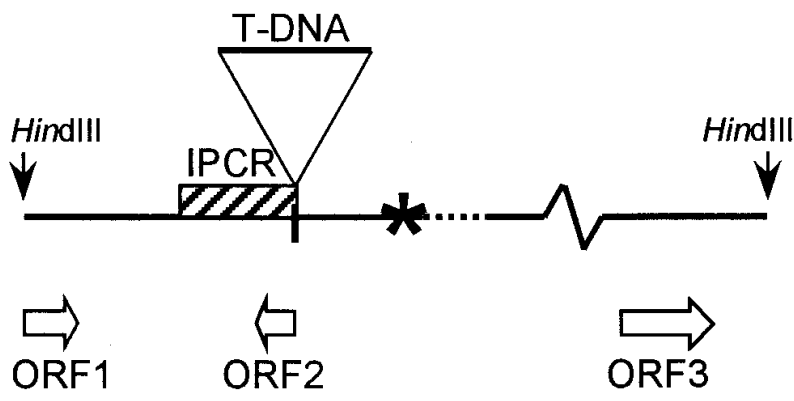

Fig. 1. Diagram of the genomic regions flanking the T-DNA integration site. A 6.6-kb HindIII fragment was subcloned from BACH15. Approximately $6.2 \mathrm{~kb}$ was sequenced (GenBank accession numbers AF018069 and AF018070); a 0.4-kb region that has so far proved recalcitrant to sequencing is shown as a dotted line. The T-DNA insertion site in dm3t524, 800 bp of lettuce DNA contained in the original inverse polymerase chain reaction product (hatched box above the line), and a 50-bp region that is absent in dm3t524 (black rectangle below the line) are shown. Opening reading frames (ORF1, ORF2, ORF3) are indicated as arrows. The position of microsatellite marker MSAT15-34 is marked by *. The 3.5-kb T-DNA insertion and the segment to the right of the 0.5 -kb break are not drawn to scale.

Table 1. Oligonucleotide primers used for polymerase chain reaction (PCR)

\begin{tabular}{|c|c|c|c|}
\hline Purpose and origin & Designation & Sequence $\left(5^{\prime}\right.$ to $\left.3^{\prime}\right)$ & Reference \\
\hline \multicolumn{4}{|l|}{ Detection of $A c$ transposase } \\
\hline $35 \mathrm{~S} 5^{\prime}$ & $\mathrm{C} 13$ & ATCTCCACTGACGTAAGGGATGACG & \multirow[t]{2}{*}{ Yang et al. 1993a } \\
\hline Ac $5^{\prime}$ & $\mathrm{J}$ & CCTCTCGGCCTCAGCTGTTGATGATGA & \\
\hline \multicolumn{4}{|l|}{ Detection of $D s$} \\
\hline Ac $5^{\prime}$ & D1 & AGTCGGAGAGAGCAGGCGAGAGCGA & \multirow[t]{2}{*}{ Yang et al. 1993a } \\
\hline Ac $5^{\prime}$ & D4 & ACTGCAATATGGCCAGCTCCAACTC & \\
\hline \multicolumn{4}{|l|}{ Detection of $D s$ donor site } \\
\hline AadA & $\mathrm{C} 14$ & AGTTGAGTCGATACTTCGGCGATCAC & \multirow[t]{2}{*}{ Yang et al. 1993a } \\
\hline Nos $5^{\prime}$ & $\mathrm{C} 15$ & GCGGGTTTCTGGAGTTTAATGAGCTAAG & \\
\hline \multirow{4}{*}{ Inverse PCR (IPCR) of T-DNA right border } & $\mathrm{C} 31$ & GGGGCATCGCACCGGTGAGTAA & \multirow{4}{*}{ Thomas et al. 1994} \\
\hline & C32 & TTGTGGGCCTGTGGTCTCAAGATG & \\
\hline & D6 & GCGGGTTTCTGGAGTTTAATGAGCTAAG & \\
\hline & D57 & ATACGTCAGAAACCATTATTGCGCG & \\
\hline \multirow[t]{4}{*}{ IPCR of T-DNA left border } & D14 & AGGTAATGGGCTACACTGAATTGG & \multirow[t]{4}{*}{ Thomas et al. 1994} \\
\hline & D43 & CGCATAATCTCAGACCAATCTGAAG & \\
\hline & D58 & GCTTGGGTCCACATTTCGCACGTAG & \\
\hline & D59 & GGCAAACAGGAATGCTCAAGCCAGG & \\
\hline \multicolumn{4}{|l|}{ Amplification of lettuce genomic DNA } \\
\hline \multirow[t]{2}{*}{ 390-bp genomic fragment } & $\mathrm{D} 23 \mathrm{~A}$ & GTCACCTGTGCACATGT & \\
\hline & D36B & CCAAGTCAACAAAAGAG & \\
\hline \multirow[t]{2}{*}{ 660-bp genomic fragment } & $\mathrm{D} 23 \mathrm{~A}$ & GTCACCTGTGCACATGT & \\
\hline & D36D & GAGTCACCAAATTCAAAG & \\
\hline \multirow[t]{2}{*}{ T-DNA integration site } & KSF1 & GGAAAGTAGCGGAACGGGGA & \\
\hline & KSR2 & GAGGGTGATGTTGTGCGGGA & \\
\hline \multicolumn{4}{|l|}{ Amplification of microsatellite marker } \\
\hline BACH15 & MSAT15-34F & GTATCACАТСССАААСТСТC & \\
\hline BACH15 & MSAT15-34R & GCAAACAACAGTTGAACTGCC & \\
\hline
\end{tabular}


allow us to identify the copy of the IPCR sequence that was tagged in dm3t524.

The BAC clones could be distinguished on the basis of restriction fragment profiles and the size of an adjacent microsatellite marker. BAC15H differed from the others in restriction patterns with EcoRV, AseI, and ScaI (data not shown). The other three BAC clones had identical restriction enzyme restriction patterns with these enzymes. However, these BACs have currently been placed in two different contigs on the basis of AFLP fingerprints and their hybridization patterns to a molecular marker, AC15 (B. Meyers, unpublished). Sequencing of the genomic region flanking the T-DNA identified a compound microsatellite sequence, $(\mathrm{CA})_{9} \mathrm{~T}(\mathrm{AG})_{15}(\mathrm{AAG})_{3}$ $(\mathrm{AG})_{5}, 864 \mathrm{bp}$ from the T-DNA integration site. This provided a highly informative marker that distinguished three classes of BAC clones (Fig. 4). BACH15 and BACH18 each had unique microsatellite array sizes; BACH19 and BACE42, which seem to be overlapping clones in the same genomic contig (B. Meyers, unpublished) contain the same size of microsatellite array. The microsatellite array size in BACH15 was absent in all of the Dm3 deletion mutants (Fig. 4). Therefore, BACH15 was likely to contain the copy of the IPCR $_{800}$ sequence most closely linked to $\mathrm{Dm} 3$.

\section{Characterization of the T-DNA insertion site.}

A 6.6-kb HindIII fragment containing the IPCR $_{800}$ sequence was subcloned from BACH15 and approximately $6.2 \mathrm{~kb}$ was sequenced (Fig. 1). PCR with primers KSF1 and KSR1, designed from sequences of the $6.6-\mathrm{kb}$ and $\mathrm{IPCR}_{800}$ fragments (Table 1), resulted in amplification of a 400-bp fragment containing the wild-type insertion site from Diana and an approximately 4-kb fragment containing the insertion site plus a truncated T-DNA from dm3t524. Presence of the T-DNA in the amplified product was confirmed by hybridization with the AadA gene. Comparison of the genomic sequences flanking the T-DNA insertion site in dm3t524 with those of wild-type cv. Diana showed that about 50 bp of lettuce DNA was missing in $\mathrm{dm} 3 \mathrm{t} 524$. Lettuce sequences were adjacent to the NptII gene; the left border of the T-DNA was missing. This data is consistent with the disparity in sizes of the restriction fragments detected on Southern blots (Fig. 2) and with the lack of IPCR products derived from primers specific for the left border. The selection of transgenic lettuce lines containing pSLJ3621 was made with spectinomycin rather than kanamycin (Yang et al. 1993a); therefore, a T-DNA lacking a functional NptII gene was possible in dm3t524.

\section{T-DNA insertion has interrupted a deduced ORF.}

The nucleotide sequence of the IPCR product and of the wild-type sequence from the HindIII BAC subclone indicated that the T-DNA insertion occurred within a putative 92 aminoacid open reading frame (ORF), designated ORF2 (Fig. 1). ORF2 showed similarity $\left[P_{(\mathrm{N})}=5.6 \times \mathrm{e}^{-7}\right]$ to zinc finger transcription factors from various eukaryotes, including Droso-

\section{A IPCR}

\section{B Nos:AadA}

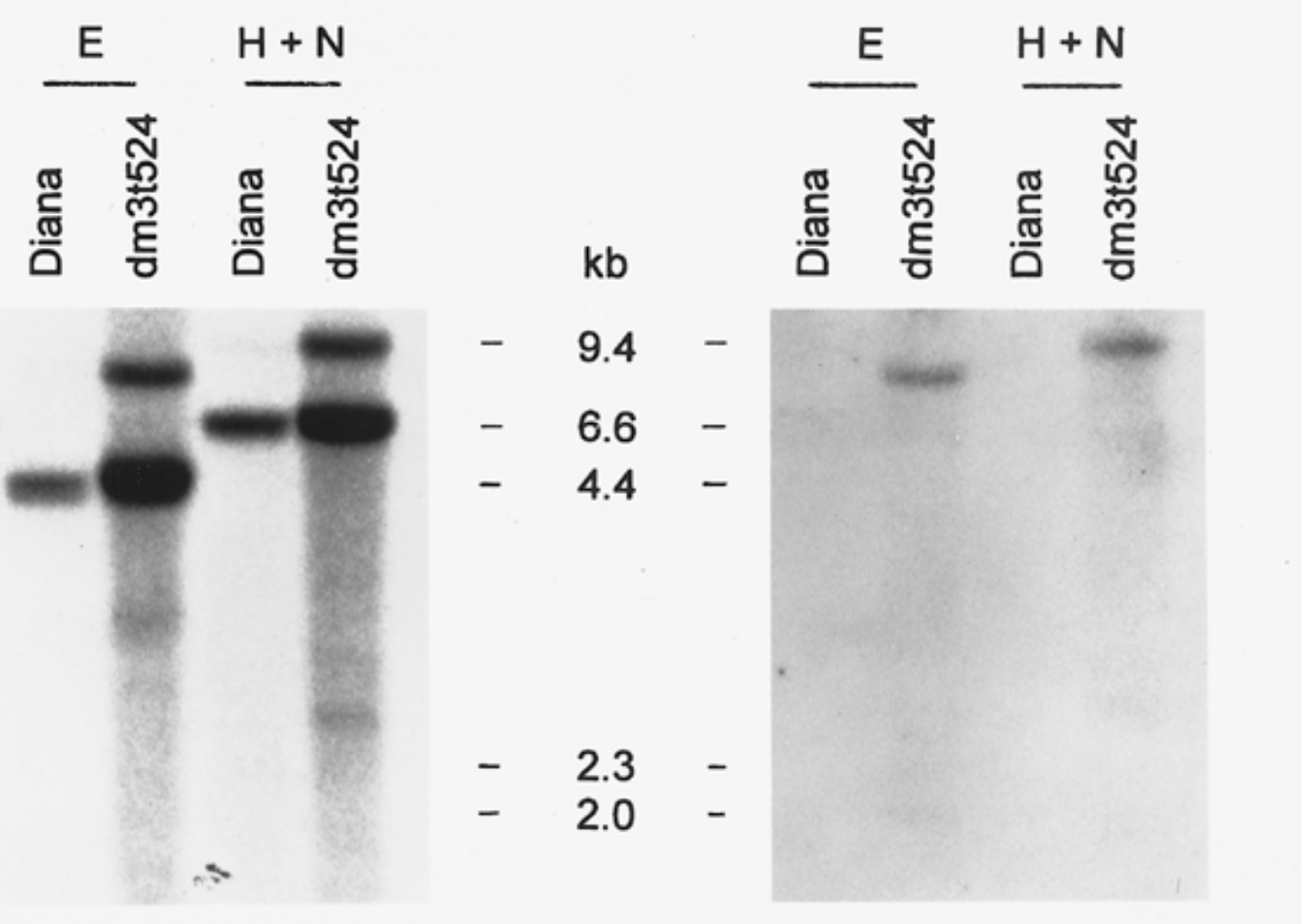

Fig. 2. Integration of a partial T-DNA results in an increased fragment size in a multigene family. Southern blots of genomic DNA from wild-type Diana (D) and dm3t524 were probed with a 390-bp genomic fragment adjacent to the integration site (A) or with a T-DNA-specific probe, Nos:AadA (B). Approximately 2 to $5 \mu \mathrm{g}$ of Diana DNA and $5 \mu \mathrm{g}$ of dm3t524 DNA were digested with either EcoRI (E) or HindIII + NotI $(\mathrm{H}+\mathrm{N})$. After hybridization with $\mathrm{IPCR}_{800}$, the membrane was stripped and re-probed with Nos:AadA. 
phila and yeast in a BLASTX search (Altschul et al. 1990). A second ORF of 138 deduced amino acids, designated ORF1, started at the beginning of the subcloned $6.6-\mathrm{kb}$ fragment in the reverse direction to ORF2 and terminated $1.58 \mathrm{~kb}$ from the T-DNA integration site (Fig. 1). A third ORF (ORF3) of 250 amino acids started $3.1 \mathrm{~kb}$ away from the T-DNA integration site and was oriented in the same direction as ORF2. No significant similarity was detected, using BLASTX (Altschul et al. 1990), between ORFs 1 or 3 and any sequences in GenBank. There were four other shorter ORFs within the $6.2-\mathrm{kb}$ nucleotide sequence. No motifs, such as nucleotide-binding domains, obvious leucine-rich repeats, or domains conserved in protein kinases, that are present in disease resistance genes from other plants (Staskawicz et al. 1995; Bent 1996), were evident in the 6.2-kb sequence. Much of the sequence was AT rich.

The possibility that $D m 3$ encoded a zinc finger protein as new class of resistance genes led to attempts to identify a cDNA or transcript. However, Northern (RNA) blots, cDNA library screens, and rapid amplification of cDNA ends (RACE) failed to identify transcripts corresponding to ORF2. PolyA RNA from cv. Diana and related cultivars did not hybridize to either $\mathrm{IPCR}_{1200}$ or BAC clones carrying wild-type copies of the 6.6-kb HindIII fragment. cDNA clones $(1.4 \times$ $10^{6}$ ) from a cv. Diana leaf mRNA library were screened under various stringencies with a combination of BAC clones as probes. These clones carried copies of the 6.6-kb HindIII fragment (Fig. 1). None of the probe combinations showed significant and reproducible hybridization. The cDNA library and leaf RNA were also used for 5' RACE and 3' RACE with ORF2-specific primers. No identifiable amplification products were obtained. Therefore, ORF 1, ORF2, and ORF3 encode low-abundance transcripts or are not transcribed. These experiments also indicated that no other abundant transcripts are encoded by BACH15.

\section{Transgenic complementation experiments with the region flanking the T-DNA insertion site.}

BACH15 contained the microsatellite array that was missing in all the deletion mutants; therefore, it was judged to be the most likely of the $\mathrm{IPCR}_{800}$-containing BAC clones to contain Dm3 (see above, Fig. 4). Overlapping 11-, 16and $27-\mathrm{kb}$ fragments of BACH15, each containing the complete $6.6-\mathrm{kb}$ HindIII fragment with the wild-type T-DNA insertion site, were introduced by Agrobacterium-mediated transformation into cv. Cobham Green (no known Dm genes) and two EMS-induced mutants of $D m 3$ in cv. Diana (R. Arroyo-Garcia, unpublished). $\mathrm{T}_{2}$ families from 27 independent transgenic plants were tested for resistance to Bremia lactucae. None of the families showed resistance to the isolate IM25P11 (Avr3). The EMS-induced mutants were resistant and Cobham Green was susceptible to the three other isolates (CG1, R60, C83M47) as expected. Therefore, either T-DNA insertion had occurred close by but not within $D m 3$, or $D m 3$ extends beyond the segments of genomic DNA tested.

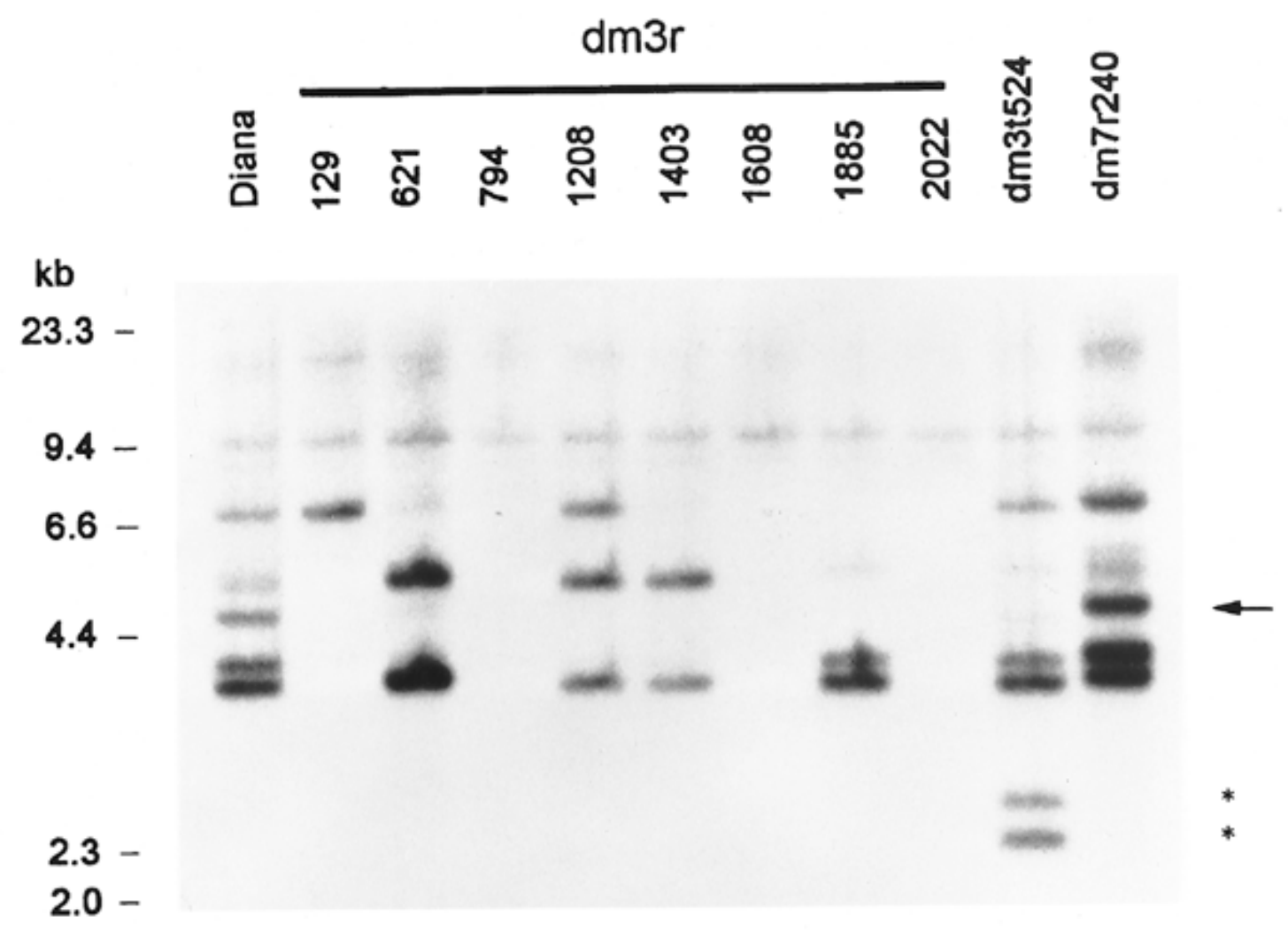

Fig. 3. The T-DNA integrated into a fragment that is missing in all $D m 3$ deletion mutants. Southern blot of genomic DNA from wild-type Diana (D) and various $D m$ mutants was probed with IPCR $_{1200}$. Each lane contains about 2.5 to $5 \mu \mathrm{g}$ of AccI-digested DNA. A 4.8-kb band (arrow) is present in both wild-type Diana and a $D m 7$ mutant (dm7r240), but is missing in all the Dm3 deletion mutants (dm3r129 to 2022). In dm3t524, two novel bands (*) are observed due to an AccI site in the T-DNA. 


\section{DISCUSSION}

The dm3t524 mutant, a transgenic line of lettuce that has lost Dm3 activity, has provided a more detailed understanding of the organization and complexity of the $D m 3$ region. It led to the identification of molecular markers, including a regionspecific microsatellite marker, very closely linked to Dm3. These markers have been useful for the isolation and characterization of BAC clones in the Dm3 region (B. Meyers, Z. Zhang, K. Shen, and R. Michelmore, unpublished).

The T-DNA inserted either in or very close to $D m 3$. The T-

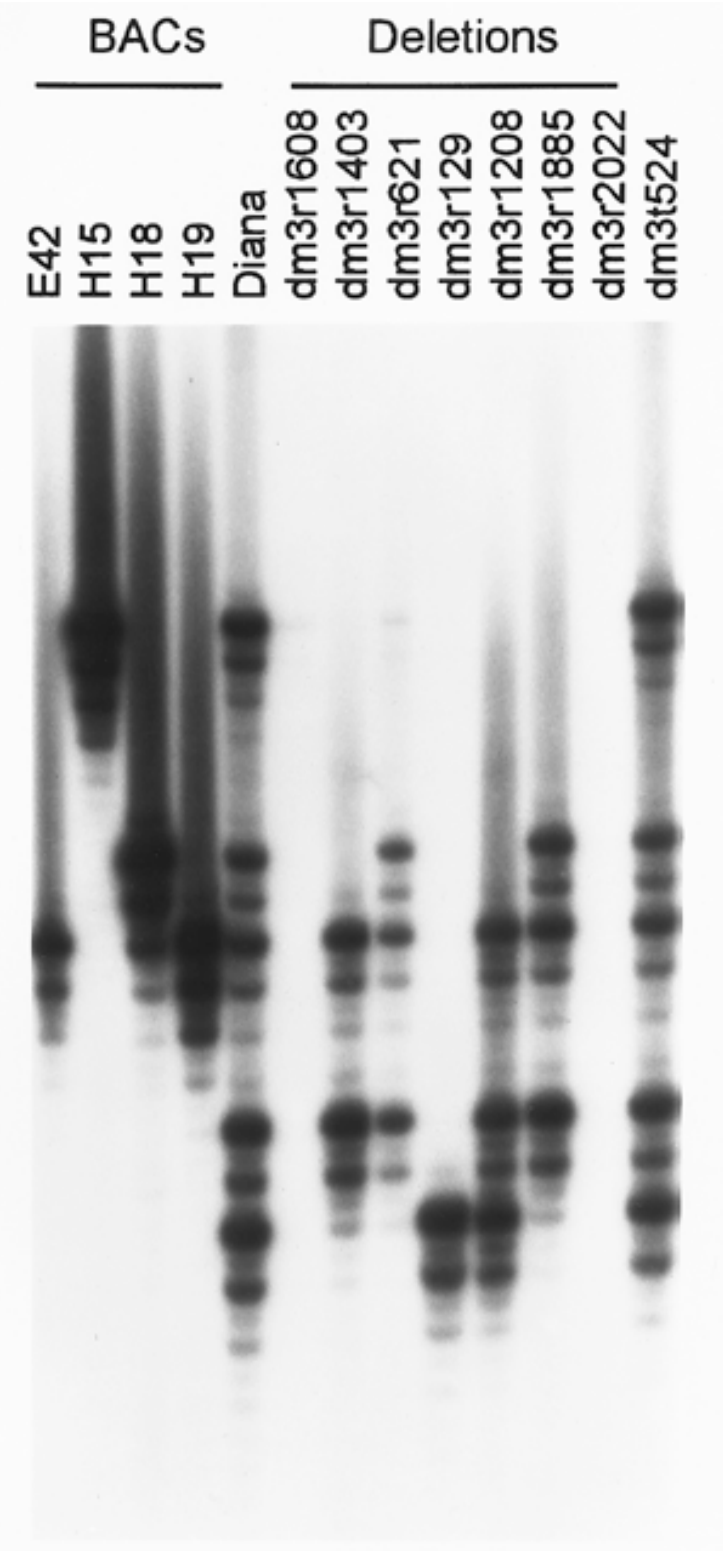

Fig. 4. A microsatellite marker $864 \mathrm{bp}$ from the T-DNA integration site detects several loci that are tightly clustered around $\mathrm{Dm} 3$ and distinguishes bacterial artificial chromosome (BAC) clones from the region. Microsatellite marker MSAT15-34 was amplified from genomic DNA of Diana (D), seven deletion mutants of Dm3, dm3t524, and four BAC clones from the Dm3 region. Each BAC clone contained a single array size. Different deletion mutants lacked different array sizes. The largest array that was missing in all deletion mutants was present in BACH15.
DNA originally contained a $D s$ element that had excised and been lost from the genome; it is impossible to determine if $D s$ excised, re-integrated close by, and then re-excised prior to being lost. Therefore, the mutation might have resulted from either T-DNA insertion into the $D m 3$ gene or from insertion of $D s$ into Dm3 and subsequent excision, leaving a "footprint" of approximately 8 bases that is difficult to locate. Such a footprint would result in a frameshift mutation and loss of $D m 3$ activity.

Transcript analyses provided no indications of gene activity at the site of T-DNA insertion. Absence of detectable transcripts of ORF1, ORF2, or ORF3 in multiple experiments suggests that they are either rare or are not transcribed in the leaf. Alternatively, the AT-rich nature of the IPCR product and flanking sequences indicates that much of the 6.6-kb fragment sequenced could be part of an intron. Several consensus sequences for GT-AG splice junctions are present throughout the HindIII fragment. Insertion of the T-DNA would prevent correct processing and cause a loss of $D m 3$ activity. In this case, ORF2 is coincidental or is a remnant of genome evolution. It is unlikely that the whole gene is represented by the 6.6-kb region sequenced. ORF1 was located at one end of the region sequenced and lacked an ATG at the beginning; therefore, it could be part of a larger ORF. However, there were no sequence motifs that indicated a function for ORF1 or ORF3.

Transgenic complementation experiments failed to restore $D m 3$ activity. Therefore, either the T-DNA did not integrate into $D m 3$ or the regions tested are not sufficient to confer Dm3 activity. To determine if the $\mathrm{IPCR}_{800}$ sequence or the 6.6$\mathrm{kb}$ HindIII fragment is actually a portion of Dm3, or is necessary for Dm3 activity, requires more extensive analysis of ORFs in the region and complementation with additional segments of genomic DNA.

\section{MATERIALS AND METHODS}

Plant material and genetic stocks.

Transformation procedures were as described previously (Michelmore et al. 1987). Cotyledons or leaf pieces of cv. Diana were sequentially co-cultivated first with Agrobacterium tumefaciens carrying Ti plasmid pSLJ3232, encoding CaMV $35 \mathrm{~S}$ promoter:Ac transposase, followed by a second cocultivation with A. tumefaciens carrying Ti plasmid pSLJ3621, encoding Ds (Yang et al. 1993a). Fungal isolates C83M47 (avirulent on Dm1), IM25P11 (avirulent on Dm3), R60 (avirulent on $D m 7$ ), and CG1 (avirulent on $D m 8$ ), and screens for loss of $\mathrm{Dm}$ activity in $\mathrm{R}_{2}$ populations were as described previously (Okubara et al. 1994). Eight Dm3 deletion mutants, two spontaneous mutants, and five EMS mutants were used for allelism tests (Table 2).

\section{Analysis for $A c, D s$, and molecular markers in the $D m 3$ region.}

DNA from $\mathrm{R}_{2}$ and $\mathrm{R}_{3}$ seedlings was analyzed by PCR for the presence of maize $A c$ transposase sequences, $D s$, the $D s$ empty donor site (Yang et al. 1993a), and molecular markers in the Dm3 region as described (Paran and Michelmore 1993). Oligonucleotide primers used for PCR are listed in Table 1.

\section{Isolation of dm3t524 genomic DNA flanking the T-DNA} and of the corresponding sequence from wild-type Diana.

Lettuce genomic sequences that flanked the T-DNA inser- 
tion site in dm3t524 were isolated initially by IPCR. Nuclear DNA was digested with BamHI and either $B c l \mathrm{I}, B g l \mathrm{II}$, or $B s t$ YI prior to ligation. Nested primers specific to the right and left borders are shown in Table 1. PCR products obtained from dm3t524 but not from wild-type Diana were inserted into the EcoRV site of pBSKII- (Stratagene, La Jolla, CA). To obtain additional sequences flanking the T-DNA insertion site, genomic DNA from wild-type Diana and from dm3t524 was amplified with primers designed from the 1,200-bp IPCR product flanking the T-DNA, obtained above. A 1-kb PCR product from dm3t524, containing a portion of pSLJ3621 plus adjacent lettuce DNA, was cloned in pAMP1 (Gibco BRL, Gaithersburg, MD).

\section{Analysis of $\mathrm{Dm} 3$ mutants by hybridization to IPCR probes in Southern blots.}

From 2.5 to $5 \mu \mathrm{g}$ of DNA from cv. Diana and the $\mathrm{Dm} 3 \mathrm{mu}-$ tants was digested with restriction enzymes, then transferred to Zeta Probe nylon membranes (BioRad, Hercules, CA) and hybridized to the 1,200-bp IPCR product (including a portion of the T-DNA right border and $800 \mathrm{bp}$ of flanking lettuce DNA) or 390- or 660-bp fragments (containing only lettuce sequences) amplified by PCR from the cloned $\mathrm{IPCR}_{1200}$ product. Probes were radiolabeled with the Multiprime DNA Labeling System (Amersham, Arlington Heights. IL) to specific activities of 2 to $4 \times 10^{6} \mathrm{cpm}$ of ${ }^{32} \mathrm{P}$ per ng of DNA. Hybridization was carried out in $50 \%$ formamide.

To estimate the number of copies of the IPCR sequence in the lettuce genome, a Southern blot of dm3t524, digested with either EcoRI or HindIII, was hybridized with the 1,200- or 660-bp IPCR fragments. A 580-bp probe consisting of the nopaline synthase (Nos) promoter and a portion of AadA gene for resistance to spectinomycin was used for detection of TDNA-associated sequences. Radiolabel that hybridized to both T-DNA-tagged and untagged copies under high-stringency conditions was quantified with a Fujix BAS 1000 phosphoimager. Quantitation was carried out by scanning lanes and measuring areas under peaks (Profile, MACBAS 2.0, Fujix, Stamford, CT), or by the direct counting of pixels in defined areas (Quant, Fujix).

Table 2. Mutants of $D m 3$ used for allelism tests with mutant dm3t524

\begin{tabular}{|c|c|c|c|}
\hline Designation & Inducing agent & Flanking makers & $\begin{array}{l}\text { Previous } \\
\text { designation }\end{array}$ \\
\hline dm3r129 & Fast neutron & Some missing & $\mathrm{dm} 3 \mathrm{a}$ \\
\hline dm3r621 & Fast neutron & Some missing & $\mathrm{dm} 3 \mathrm{~b}$ \\
\hline dm3r794 & Fast neutron & Some missing & $\mathrm{dm} 3 \mathrm{c}$ \\
\hline dm3r1208 & Fast neutron & Some missing & $\mathrm{dm} 3 \mathrm{~d}$ \\
\hline $\mathrm{dm} 3 \mathrm{r} 1403$ & Fast neutron & Some missing & $\mathrm{dm} 3 \mathrm{e}$ \\
\hline $\mathrm{dm} 3 \mathrm{r} 1608$ & Fast neutron & Some missing & $\mathrm{dm} 3 \mathrm{f}$ \\
\hline $\mathrm{dm} 3 \mathrm{r} 1885$ & Fast neutron & Some missing & $\mathrm{dm} 3 \mathrm{~h}$ \\
\hline dm3r2022 & Fast neutron & Some missing & $\mathrm{dm} 3 \mathrm{i}$ \\
\hline $\mathrm{dm} 3 \mathrm{e} 310$ & $\mathrm{EMS}^{\mathrm{b}}$ & All present & \\
\hline dm3e407 & EMS & All present & \\
\hline $\mathrm{dm} 3 \mathrm{e} 1368$ & EMS & All present & \\
\hline dm3e1534 & EMS & All present & \\
\hline dm3e1591 & EMS & All present & \\
\hline $\mathrm{dm} 3 \mathrm{~s} 218$ & Spontaneous & Some missing & \\
\hline dm3s1977 & Spontaneous & All present & \\
\hline
\end{tabular}

${ }^{a}$ The names of mutants reported previously (Okubara et al. 1994; Anderson et al. 1996) have been revised.

${ }^{\mathrm{b}}$ Ethyl methanesulfonate.

\section{BAC clones containing IPCR sequences.}

Over 50,000 BAC clones from two different libraries of cv. Diana, representing approximately two genome-equivalents (Frijters et al. 1997), were screened with the IPCR 660-bp probe. Four positive BAC clones, designated BACH15, BACH18, BACH19, and BACE42, were treated with AccI, Ase I, EcoRV, or ScaI, diagnostic for different copies of the IPCR sequence. HindIII fragments of about $6.6 \mathrm{~kb}$ from $\mathrm{BACH} 15, \mathrm{BACH} 18$, and $\mathrm{BACH} 19$ that hybridized to the IPCR 660-bp probe in Southern blots were inserted into a lowcopy plasmid vector, pBeloBAC11 (Shizuya et al. 1992).

BAC clones were distinguished with a microsatellite sequence identified adjacent to the T-DNA integration site. Primers were end labeled with $\gamma^{32} \mathrm{P}$ ATP. Microsatellites were amplified with Taq DNA polymerase, 10 pmol of each primer (Table 1), $50 \mathrm{ng}$ of genomic DNA, and $5 \mathrm{mM}$ dNTPs in a reaction volume of $20 \mu \mathrm{l}$ for 30 cycles of $30 \mathrm{~s}$ at $94^{\circ} \mathrm{C}, 30 \mathrm{~s}$ at $60^{\circ} \mathrm{C}$, and $1 \mathrm{~min}$ at $72^{\circ} \mathrm{C}$. Reaction products were separated on a $8 \%$ denaturing polyacrylamide gel.

\section{Nucleotide sequencing and database searches.}

Portions of the HindIII subclones derived from BAC clones carrying the IPCR sequence and clones of amplified genomic DNA from Diana and dm3t524 were sequenced with an ABI373 Automated DNA Sequencer (Applied Biosystems, Foster City, CA). Primers used for sequencing are listed in Table 1. Nucleotide and deduced amino acid sequences were analyzed for similarities to known sequences in GenBank with BLASTX (Altschul et al. 1990).

\section{Constructs for transgenic complementation.}

DNA from BACH15 was partially digested with HindIII, size fractionated by gel electrophoresis, and cloned into the binary vector pCGN1557 (McBride and Summerfelt 1990). Clones that contained fragments with the T-DNA insertion site were identified by restriction analysis and hybridization. These were introduced into lettuce by Agrobacteriummediated transformation, using previously published protocols (Michelmore et al. 1987) with Agrobacterium strain LBA4404. The presence of the insert in lettuce was confirmed by PCR with primers for NptII and growth of seedlings on kanamycin (200 mg/liter). All plants tested were transgenic. The presence of the 6.6-kb fragment was demonstrated in Cobham Green with the microsatellite marker MSAT15-34. It was not possible to test for the insert in the EMS-induced mutants of cv. Diana as they already had the wild-type sequence except presumably for a point mutation in the $D m 3$ gene. At least $50 \mathrm{~T}_{2}$ seedlings from each transgenic plant were tested for resistance to each of the following isolates: IM25P11 (avirulent on Dm3); C83M47 (avirulent on Dml); R60 (avirulent on Dm7); and CG1 (avirulent on Dm8).

\section{ACKNOWLEDGMENTS}

We thank Jonathan Jones and Bernie Carroll (Sainsbury Laboratories, Norwich, UK) for pSLJ3232, pSLJ3621, and the primers for IPCR; Adrie Frijters, Zhen Zhang, and Guo-Liang Wang for construction and screening of BAC libraries of lettuce; Dean Lavelle for automated sequencing; Oswaldo Ochoa, Harpreet Johl, Katy McCann, and Grace Salcedo for screening EMS and spontaneous mutants; Matt Kreiter and Gregg Morin for help with phosphoimaging quantitation; and Steve Scofield for insightful discussions. This work was supported by USDA 
NRICGP grant 92-37300-7547. In addition, P. A. O. was supported in part by NIH postdoctoral fellowship F32 GM14673, and R. A.-G. was supported in part by a postdoctoral fellowship from the Instituto Nacional de Investigaciones Agrarias, Spain.

\section{LITERATURE CITED}

Altschul, S. F., Gish, W., Miller, W., Myers, E. W., and Lipman, D. J. 1990. Basic local alignment search tool. J. Mol. Biol. 215:403-410.

Anderson, P. A., Okubara, P. A., Arroyo-Garcia, R., Meyers, B. C., and Michelmore, R. W. 1996. Molecular analysis of irradiation-induced and spontaneous deletion mutants at a disease resistance locus in Lactuca sativa. Mol. Gen. Genet. 251:316-325.

Bent, A. F. 1996. Plant disease resistance genes: Function meets structure. Plant Cell 8:1757-1771.

Bent, A. F., Kunkel, B. N., Dahlbeck, D., Brown, K. L., Schmidt, R. L., Giraudat, J., Leung, J. L., and Staskawicz, B. J. 1994. RPS2 of Arabidopsis thaliana: A leucine-rich repeat class of plant disease resistance genes. Science 265:1856-1860.

Farrara, B., Illott, T. W., and Michelmore, R. W. 1987. Genetic analysis of factors for resistance to downy mildew (Bremia lactucae) in lettuce (Lactuca sativa). Plant Pathol. 36:499-514.

Frijters, A. C. J., Zhang, Z., van Damme, M., Wang, G.-L., Ronald, P. C., and Michelmore, R. W. 1997. Construction of a bacterial artificial chromosome library containing large EcoRI and HindIII genomic fragments of lettuce. Theor. Appl. Genet. 94:390-399.

Grant, M. R., Godiard, L., Straube, E., Ashfield, T., Lewald, J., Sattler, A., Innes, R. W., and Dangl, J. L. 1995. Structure of the Arabidopsis $R P M 1$ gene enabling dual specificity disease resistance. Science 269: 843-846.

Johal, G. S., and Briggs, S. P. 1992. Reductase encoded by the Hml disease resistance gene in maize. Science 258:985:987.

Jones, J. A., Thomas, C. M., Hammond-Kosack, K. E., Balint-Kurti, P. J., and Jones, J. D. G. 1994. Isolation of the tomato $C f-9$ gene for resistance to Cladosporium fulvum by transposon tagging. Science 266: 789-793.

Kanazin, V., Marek, L. F., and Shoemaker, R. C. 1996. Resistance gene analogs are conserved and clustered in soybean. Proc. Natl. Acad. Sci. USA 93:11746-11750.

Kobe, B., and Deisenhofer, J. 1994. The leucine-rich repeat: A versatile binding motif. Trends Biochem. Sci. 19:415-421.

Lawrence, G. J., Finnegan, E. J., Ayliffe, M. A., and Ellis, J. G. 1995. The $L 6$ gene for flax rust resistance is related to the Arabidopsis bacterial resistance gene RPS2 and the tobacco viral resistance gene $N$. Plant Cell 7:1195-1206.

Martin, G. B., Brommonschenkel, S. H., Chunwongse, J., Frary, A., Ganal, M. W., Spivey, R., Wu, T., Earle, E. D., and Tanskley, S. D. 1993. Map-based cloning of a protein kinase gene conferring disease resistance in tomato. Science 262:1432-1436.
McBride, K. E., and Summerfelt, K. R. 1990. Improved binary vectors for Agrobacterium-mediated plant transformation. Plant Mol. Biol. 14:269-276.

Michelmore, R. W. 1995a. Isolation of disease resistance genes from crop plants. Curr. Opin. Biotechnol. 6:145-152.

Michelmore, R. W. 1995b. Molecular approaches to manipulation of disease resistance genes. Annu. Rev. Phytopathol. 15:393-427.

Michelmore, R. W., Marsh, E., Seeley, S., and Landry, B. S. 1987. Transformation of lettuce mediated by Agrobacterium tumefaciens. Plant Cell Rep. 6:439-442.

Okubara, P. A., Anderson, P. A., Ochoa, O. E., and Michelmore, R. W. 1994. Mutants of downy mildew resistance in Lactuca sativa (lettuce). Genetics 137:867-874.

Paran, I., and Michelmore, R. W. 1993. Development of reliable PCRbased markers linked to downy mildew resistance genes in lettuce. Theor. Appl. Genet. 85:985-993.

Salmeron, J. M., Barker, S. J., Carland, F. M., Mehta, A. Y., and Staskawicz, B. J. 1994. Tomato mutants altered in bacterial disease resistance provide evidence for a locus controlling pathogen recognition. Plant Cell 6:511-520.

Shizuya, H., Birren, B., Kim, U., Mancino, V., Slepak, T., Tachiiri, Y., and Simon, M. 1992. Cloning and stable maintenance of 300kilobase-pair fragments of human DNA in Escherichia coli using an F-factor-based vector. Proc. Natl. Acad. Sci. USA 89:8794-8797.

Song, W.-Y., Wang, G. L., Chen, L.-L., Kim, H.-S., Pi, L.-Y., Holsten, T., Gardner, J., Wang, B., Zhai, W.-X., Zhu, L.-H., Fauquet, C., and Ronald, P. 1995. A receptor kinase-like protein encoded by the rice disease resistance gene, Xa21. Science 270:1804-1806.

Staskawicz, B. J., Ausubel, F. M., Baker, B. J., Ellis, J. G., and Jones, J. D. G. 1995. Molecular genetics of plant disease resistance. Science 268:661-667.

Thomas, C. M., Jones, D. A., English, J. J., Caroll, B. J., Bennetzen, J. L., Harrison, K., Burbidge, A., Bishop, G. J., and Jones, J. D. G. 1994. Analysis of the chromosomal distribution of transposon-carrying TDNAs in tomato using the inverse polymerase chain reaction. Mol. Gen. Genet. 242:573-585.

Webb, G. C., and Chaplin, D. D. 1990. Genetic variability at the human tumor necrosis factor loci. J. Immunol. 145:1278-1285.

Whitham, S., Dinesh-Kumar, S. P., Choi, D., Hehl, R., Corr, C., and Baker, B. 1994. The product of the tobacco mosaic virus resistance $N$ gene: Similarity to Toll and the interleukin-1 receptor. Cell 78:1101-1115.

Yang, C.-H., Carroll, B., Scofield, S., Jones, J., and Michelmore, R. 1993a. Transactivation of $D s$ elements in plants of lettuce (Lactuca sativa). Mol. Gen. Genet 241:389-398.

Yang, C.-H., Ellis, J.G., Michelmore, R.W. 1993b. Infrequent transposition of Ac in lettuce, Lactuca sativa. Plant Mol. Biol. 22:793-805.

Yu, Y. G., Buss, G. R., and Saghai Maroof, M. A. 1996. Isolation of a superfamily of candidate disease-resistance genes in soybean based on a conserved nucleotide-binding site. Proc. Natl. Acad. Sci. USA 93:11751-11756 